\title{
Influence des caractéristiques hydrauliques des biefs sur la propagation des pointes de crue
}

\author{
Marcio Baptista $\left({ }^{1}\right)$, Claude Michel $\left({ }^{2}\right)$
}

\section{Introduction}

La propagation d'une onde de crue le long d'un cours d'eau est décrite par un système d'équations aux dérivées partielles proposé par BARRÉ dE SAINT VENANT, en 1871 (CHow, 1959).

Du fait qu'il n'y a pas de solution analytique connue, l'étude du phénomène avec les équations complètes n'a été possible qu'à partir des années 50 , avec l'avènement des ordinateurs. Aujourd'hui, même avec l'essor de l'informatique, la résolution du système d'équations reste encore un problème assez complexe et relativement fastidieux pour certaines applications. En effet, même dans les cas les plus simples, unidimensionnels, les calculs sont longs et délicats, soumis éventuellement à des difficultés numériques, et l'introduction des données est toujours laborieuse. Il demeure donc intéressant de disposer de modèles d'utilisation plus immédiate, comme on peut le constater au vu de quelques publications récentes (TINGSANCHALI et LAL, 1988), (Pridal et JAMES, 1989).

En outre, dans le domaine de l'hydrologie des milieux naturels, on doit souvent se contenter de résultats approchés, du fait de la nature incertaine des informations hydrologiques. Il est illusoire de rechercher une grande finesse dans les résultats, avec l'emploi de modèles complexes, si l'on ne dispose pas de données qui soient assez fiables. De plus, fréquemment en hydrologie, on s'intéresse seulement aux caractéristiques d'atténuation et de décalage d'une onde de crue, sa forme présentant un intérêt secondaire.

C'est ce type de problème que nous nous proposons de résoudre, au prix d'un certain nombre de simplifications, sauvegardant les principales variables et permettant d'aboutir à une méthode simple et rapide, donnant une première estimation des principaux éléments d'une propagation. On pourra ainsi dégrossir des problèmes fréquents dans la pratique, comme, par exemple, l'appréciation de l'impact sur les pointes de crues d'un aménagement de rivière, qui ferait passer le coefficient de STRICKLER d'une valeur moyenne de 25 à une valeur moyenne de 35 .

(') Ingénieur, élève-chercheur de l'Ecole nationale des Ponts et Chaussées, en stage à la division hydrologie du CEMAGREF-ANTONY.

${ }^{2}$ ) Ingénieur à la division hydrologie du CEMAGREFANTONY et au CERGRENE.

\section{Influence of the hydraulic characteristics of river ponds on the propagation of flood peaks}

In the field of the hydrology of the natural environment, it is often necessary to have to accept approximative results due to the uncertain nature of hydrological information. Moreover, very often what is only of interest are the increasing and reduction characteristics of a flood peak and its form seems to be only of secondary interest. This is the type of problem that the authors attempt to resolve, at the expense of a certain number of simplifications, holding on to the main variables and enabling us to come up with a simple rapid method which gives us an initial estimation of the main elements of a propagation. We may thus reduce the number of practical problems such as, for example, appreciation of the impact of a river development on high-water peaks which would make the Strickler coefficient go from an average value of 25 to an average value of 35 . 


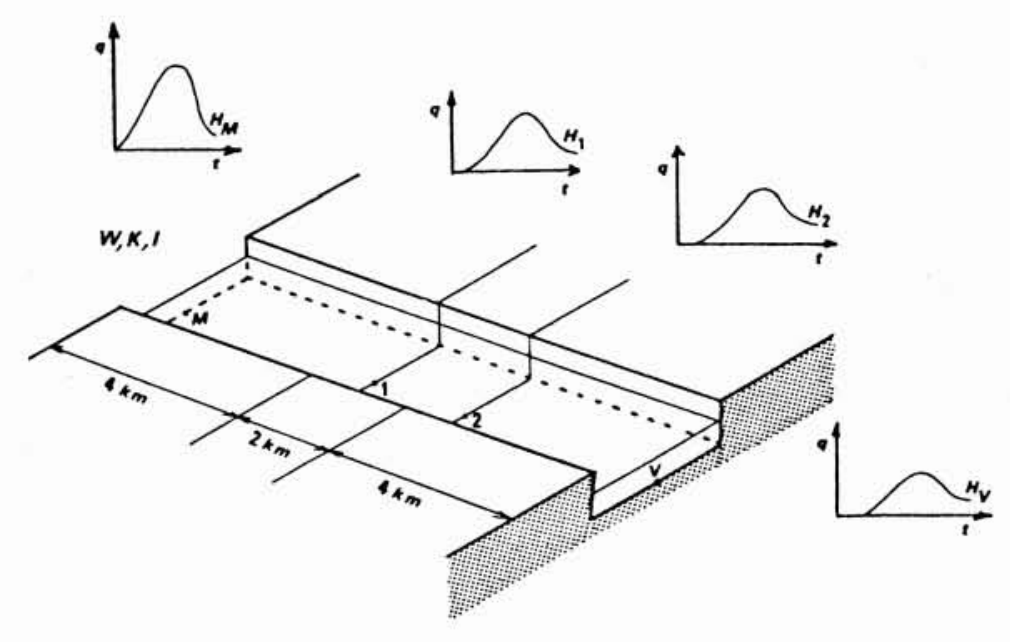

1. Schématisation du tronçon de cours d'eau et des crues considérés.
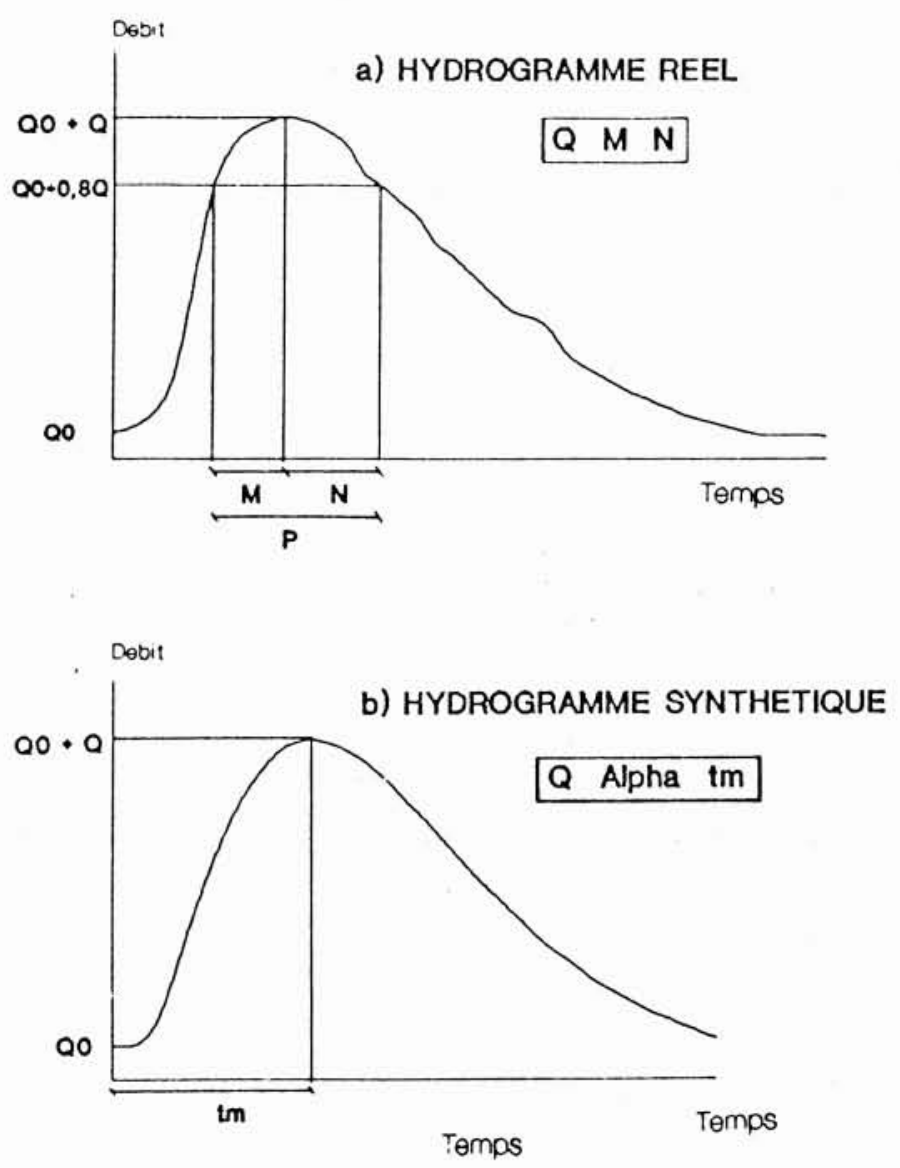

2. Hydrogramme réel et hydrogramme synthétique.

\section{Démarche adoptée}

La démarche adoptée dans l'étude est la suivante :

- simulation de la propagation d'un certain nombre de crues sur plusieurs biefs différents avec utilisation du modèle complet de SAINT VENANT;

- obtention de relations entre les variables du phénomène de propagation auxquelles on s'intéresse et les caractéristiques physiques des cours d'eau et des crues ; - proposition d'un modèle simplifié avec établissement d'un système d'équations algébriques, du fait que l'on s'intéresse seulement à la pointe de débit.

Avant d'exposer la mise en œuvre de cette démarche, il nous faut préciser notre description des biefs et des crues, ainsi que le modèle utilisé pour la résolution numérique du système de SAINT Venant complet. Ces différents éléments sont décrits ci-après.

\subsection{Le modèle mathématique}

Le modèle mathématique utilisé pour la résolution du système de SAINT VENANT est fondé sur un schéma numérique de différences finies implicite : le "schéma à 4 points de PreismanN ", décrit par Cunge, Holly et VERWEY (1980).

La résolution de ce schéma numérique est faite par la méthode du "double balayage" (Pochat, 1980). Sachant que l'on a adopté la valeur du paramètre d'interpolation $\theta$, caractéristique du schéma numérique, comme égale à $3 / 4$, le schéma est inconditionnellement stable; le seul problème est celui de la précision des résultats, qui est fonction des caractéristiques choisies pour la discrétisation: le pas de temps, le pas d'espace, la valeur de $\theta$ et le nombre de points représentatifs de la crue (Aвbot, 1979), (CUNGe, Holly et Verwey, 1980) et (TuCCI, 1986). Les vérifications effectuées, avec les données utilisées, selon les indications de Аввот (1979), nous conduisent à des erreurs inférieures à $5 \%$, soit pour la vitesse, soit pour l'atténuation.

\subsection{Les biefs}

En ce qui concerne les biefs, on a adopté une schématisation par une section rectangulaire large, caractérisée par la largeur $W$, la rugosité et la pente. Si l'on travaille avec des débits par unité de largeur, celle-ci n'apparaît plus de façon explicite et un bief est caractérisé par le couple rugosité-pente.

Pour permettre la généralisation des résultats, on a opté pour une variation assez large et représentative des valeurs de ces paramètres. On a utilisé la gamme suivante des valeurs de la rugosité de STRICKLER $(K)$ et de la pente $(I)$ :

- $K: 15,30$ et 50 ;

- I: 0,00002, 0,00010, 0,00050, et 0,00200 .

La combinaison de ces deux paramètres nous permet donc de traiter 12 biefs, hydrauliquement assez différents et représentatifs de cours d'eau et canaux réels.

Dans les biefs considérés dans notre étude, on s'intéresse seulement à un tronçon central de $2 \mathrm{~km}$ (point 1 au point 2 sur la fig. 1). On encadre ce tronçon central par 
deux tronçons de $4 \mathrm{~km}$, ce qui doit permettre de diminuer l'influence des conditions aux limites, à l'amont et à l'aval.

Le choix de la longueur du tronçon étudié de $2 \mathrm{~km}$ résulte d'un compromis entre deux contraintes: d'une part, on doit traiter un tronçon assez court pour diminuer le temps de calcul des simulations et, d'autre part, assez long pour éviter des problèmes d'imprécision numérique, qui pourraient être significatifs en cas de variations trop faibles des grandeurs qui nous intéressent.

\subsection{Les hydrogrammes}

Selon une démarche similaire à celle adoptée pour les biefs, il nous faut aussi une représentation simplifiée des hydrogrammes à propager, tout en gardant la bonne représentativité des hydrogrammes réels. On a choisi d'adopter une représentation analytique, qui doit présenter certaines caractéristiques considérées comme pertinentes pour l'objectif de l'étude, telles que la dérivabilité, la souplesse, etc... Plusieurs expressions analytiques existent dans la littérature. On pourrait prendre, entre autres, l'hydrogramme unitaire du modèle HAYAMI, l'hydrogramme-type de NASH, ... Pour des raisons de faisabilité des traitements ultérieurs, il faut se limiter à 3 paramètres descriptifs :

- un paramètre d'échelle sur les durées;

— un paramètre d'échelle sur les débits ;

- un paramètre de forme.

L'hydrogramme de NASH est séduisant, à première vue, mais les premiers essais ont montré que son paramètre de forme n'apporte pas la souplesse à l'endroit souhaitable pour décrire de façon satisfaisante la déformation de la crue au cours de la propagation. Pour cette raison nous avons retenu l'expression (1) légèrement différente :

$$
q=Q\left(t / t_{m}\right)^{2} \exp \left\{(2 / \alpha)\left[1-\left(t / t_{m}\right)^{\alpha}\right]\right\}+Q_{0}
$$

où :

$q \quad:$ débit fonction du temps $t$;

$Q \quad$ : débit de pointe mesuré au-dessus du débit de base ;

$t_{m} \quad:$ temps de montée ;

$\alpha \quad$ : paramètre caractéristique de la forme de la crue ;

$Q_{0} \quad$ : débit de base et débit initial.

L'hydrogramme obtenu avec cette expression apparaît sur la figure $2 b$ :

Les paramètres $t_{m}$ et $\alpha$ de l'hydrogramme synthétique seront calés à partir des paramètres $M$ et $N$ des crues réelles, qui correspondent à la durée de montée et de décrue pour la partie de l'hydrogramme dépassant $80 \%$ du débit de pointe, comme on le voit sur la figure 2. Le paramètre $P$ désigne la durée $M+N$, durée totale de dépassement de $Q_{0}+0,8 Q$. Si $W$ est la largeur du bief, seul $q / W$ intervient dans les calculs, $W$ jouant un rôle sans influence de facteur d'échelle.

La durée, $P=M+N$, correspond à la valeur de $t_{2}-t_{1}, t_{1}$ et $t_{2}$ étant les racines de l'équation (2):

$$
\left(t / t_{m}\right)^{2} \exp \left\{(2 / \alpha)\left[1-\left(t / t_{m}\right)^{\alpha}\right]\right\}=0,8 .
$$

La résolution numérique de ces expressions pour un grand nombre de valeurs de $\alpha$ nous a permis d'établir les expressions approchées (3) et (4) :

$$
\begin{aligned}
\alpha & =2,86(M / N)^{3,2} \\
t_{m} & =1,04(M+N) \alpha^{0,53} .
\end{aligned}
$$

Les expressions (3) et (4) nous permettent donc de passer des données facilement repérables, $M$ et $N$, aux paramètres $\alpha$ et $t_{m}$ utilisés dans le modèle.

De façon à permettre d'étudier convenablement l'influence des différentes caractéristiques de la crue, on a retenu la gamme suivante de variation des 3 paramètres de l'expression (1):

$$
\begin{array}{ll}
Q / W: & 1,0,2,0 \text { et } 4,0 \mathrm{~m}^{2} \mathrm{~s}^{-1} ; \\
t_{m} & : 5400,10800 \text { et } 21600 \mathrm{~s} ; \\
\alpha & : 0,75,1,00 \text { et } 2,00 .
\end{array}
$$

On a donc traité 27 hydrogrammes différents, correspondant à la combinaison de ces 3 paramètres. Ces hydrogrammes concernent l'hydrogramme " $H_{M}$ " de la figure 1. Les hydrogrammes effectivement pris en compte sont les hydrogrammes " $H_{1}$ " et " $H_{2}$ ", peu influencés par les conditions aux limites.

Dans la figure 3 on présente, à titre d'illustration, les hydrogrammes correspondants aux trois valeurs choisies pour $\alpha$.

On peut voir que l'on traite des hydrogrammes de formes assez différentes, et que l'on peut donc considérer notre échantillon comme assez diversifié pour représenter des crues réelles.

La forme analytique traduite par l'équation (2) sera calée sur les hydrogrammes fournis par la résolution numérique des équations complètes de SAINT VENANT. Cela est indispensable pour pouvoir enchaîner des propagations sur des biefs successifs.

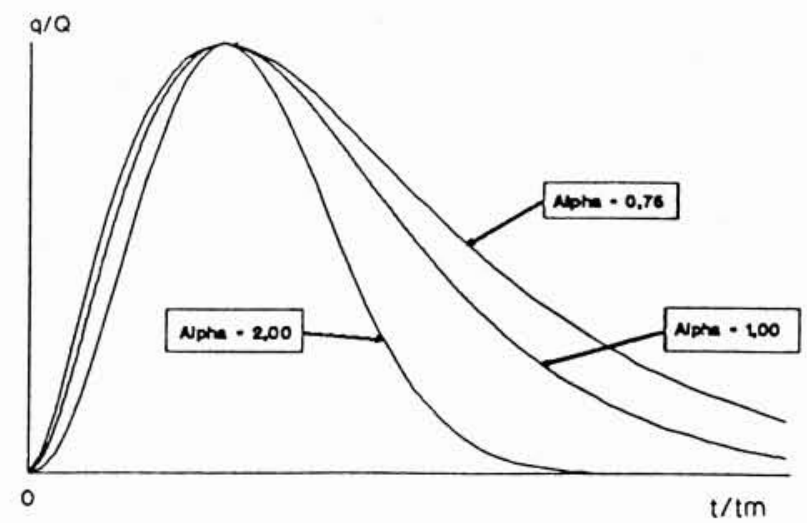

3. Hydrogrammes adoptés. 
Une autre variable que l'on pourrait prendre en compte est le débit de base. Cependant, comme déjà constaté antérieurement (CEMAGREF, 1985), une étude préalable, avec 12 crues sur 4 biefs, soit 48 situations, et des débits de base correspondant à 5,10 et $20 \%$ du débit de pointe, a mis en évidence l'absence d'influence sensible de cette variable sur les résultats du routage. Le débit de base retenu est de $10 \%$ du débit maximum direct $(Q)$.

\subsection{La condition aval}

Dans l'étude de la propagation avec les hypothèses complètes de SAINT VENANT, la condition en limite aval joue un rôle indubitable, et il faut donc essayer d'apprécier son effet dans la modélisation.

Pour ce faire, on a effectué la simulation de l'écoulement avec trois conditions aval différentes. Tout d'abord, on simule la propagation dans 324 situations (soit 27 crues et 12 biefs) avec une condition aval qui correspond à la profondeur normale en limite aval (point $V$ ) du bief, à $4 \mathrm{~km}$ de l'extrémité aval du tronçon central étudié (voir fig. l). Ensuite on a procédé à des changements de cette condition, par la création d'un rétrécissement de $20 \%$, puis d'un élargissement de même valeur pour la section aval. On a simulé ainsi 324 situations pour chacune de ces deux modifications de la condition aval, soit au total 972 simulations.

Les résultats de cette analyse ne seront pas exposés dans le cadre restreint du présent document. Nous nous bornerons à dire que la condition aval s'est révélée avoir une influence secondaire sur la propagation d'une pointe de crue et que l'on peut, en général, la négliger, si l'on cherche un ordre de grandeur de l'atténuation et du temps de transfert d'une pointe de crue.

\section{Les résultats de la simulation}

Après le traitement des différents cas décrits précédemment, on a tenté de dégager les liaisons entre les différentes variables du phénomène étudié. On décrit, dans ce qui suit, les résultats obtenus, d'abord pour la déformation de la crue occasionnée par la propagation et ensuite pour les éléments de base de la propagation.

\subsection{Les éléments de I'hydrogramme}

Pour permettre de suivre la déformation de l'hydrogramme, on a effectué des régressions sur les paramètres temps de montée et sur une variable homogène à un volume, donnée par le produit $P Q$, en ayant comme variables explicatives les différentes caractéristiques des cours d'eau et des crues.

Les régressions effectuées nous ont permis d'aboutir à des résultats très simples. En effet, comme on peut le voir, le temps de montée et le produit $P Q$ restent, grossièrement, constants après routage :

$$
t_{m 2}=t_{m 1}^{1,02}, \text { soit } t_{m 2}=t_{m 1}
$$

où $t_{m 1}$ et $t_{m 2}$ sont, respectivement, le temps de montée à l'entrée et à la sortie du tronçon central.

$$
P_{2} Q_{2}=\left(P_{1} Q_{1}\right)^{1,01} \text {, soit } P_{2} Q_{2}=P_{1} Q_{1}
$$

où $Q_{1}$ et $Q_{2}$ sont les débits de pointe, à l'entrée et à la sortie du tronçon central (1-2); $P_{1}$ et $P_{2}$ sont les durées de dépassement à l'entrée et à la sortie.

Ces résultats seront très intéressants et utiles, en permettant une simplification considérable des calculs à venir, car on considérera que $t_{m}$ et $P Q$ sont constants au cours de la propagation.

Bien que la forme de la pointe (rapport $M / N$ ) n'ait pas d'influence notable sur la propagation de la crue, les hypothèses précédentes montrent que cette forme évolue au cours de la propagation. On peut donc accessoirement évaluer cette déformation. En effet, les relations (5) et (6) jointes aux relations (3) et (4) permettent l'obtention de $M_{2}$ et $N_{2}$; on peut vérifier que les calculs conduisent aux expressions (7) et (8) :

$$
\begin{aligned}
& \frac{M_{2}}{\overline{M_{1}}}=\frac{\left(M_{1}+N_{1}\right)\left(Q_{1} / Q_{2}\right)}{M_{1}+N_{1}\left(Q_{1} / Q_{2}\right)^{0,6}} \\
& \frac{N_{2}}{N_{1}}=\frac{\left(N_{1}+M_{1}\right)\left(Q_{1} / Q_{2}\right)}{N_{1}+M_{1}\left(Q_{2} / Q_{1}\right)^{0.6}} .
\end{aligned}
$$

\subsection{Les éléments de la propagation}

\subsubsection{Modélisation proposée}

Puisque l'on s'intéresse essentiellement à la pointe de la crue et non pas à tout un hydrogramme, une variable disparaît dans notre description du phénomène et l'on doit pouvoir le représenter par de simples équations différentielles à la place des équations aux dérivées partielles.

Si $x$ représente l'abscisse de la section considérée le long du bief et si l'on désigne par $Q$ le débit maximum à l'abscisse $x$ et $T$ l'instant où débute la pointe de l'hydrogramme en cette section (cf. fig. 4), notre système d'équations sera :

$$
\begin{aligned}
& \mathrm{d} Q / \mathrm{d} x=f(K, I, \ldots) \\
& \mathrm{d} T / \mathrm{d} x=g(K, I, \ldots) .
\end{aligned}
$$

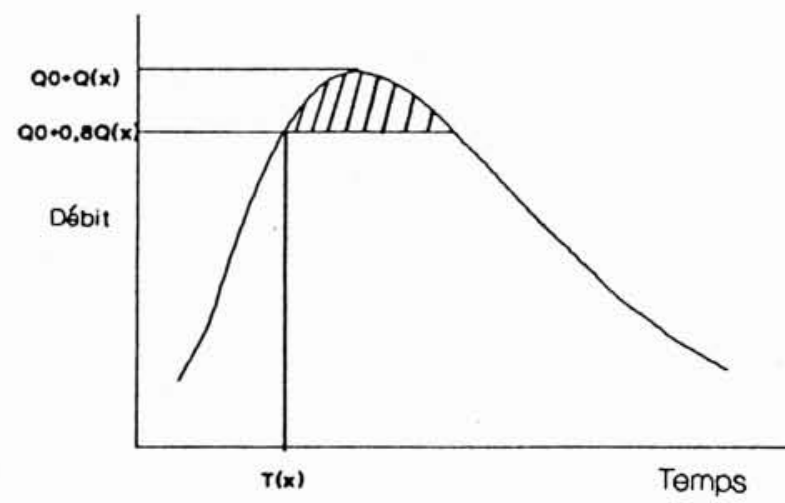

4. La pointe de la crue à la section d'abscisse $x$. 
Une difficulté apparaît du fait que les dérivées de $Q$ et $T$ ne peuvent être que déduites de rapports tels que $\Delta Q / \Delta x$ et $\Delta T / \Delta x$.

Nous avons choisi de poser a priori le modèle défini par les équations (9) et (10); d'intégrer ce modèle sur le pas d'espace correspondant au tronçon central et de caler les fonctions $f$ et $g$ pour retrouver les résultats de la simulation numérique, à savoir $\Delta Q$ et $\Delta T$.

Il reste deux grandes options dans la spécification des fonctions $f$ et $g$. Si l'on prend comme variables de ces fonctions des éléments invariants le long du bief on obtiendra un modèle rigoureusement indépendant de la longueur choisie pour le tronçon central. Le résultat obtenu conduit à des erreurs de $7 \%$ pour le débit maximum aval et de $37 \%$ pour le temps de transfert, pour une distance de $5000 \mathrm{~m}$.

Nous avons préféré un modèle plus précis, bien que moins rigoureux, conduisant à des expressions dont la validité est limitée à un pas d'espace fixe, celui du tronçon central où sont observés les valeurs $\Delta T$ et $\Delta Q$.

C'est ce modèle dont nous présentons ci-après les deux composantes: l'atténuation et la durée de propagation de la pointe.

\subsubsection{Etude de l'atténuation de la pointe}

On peut préciser un peu le modèle donné par (9) avec l'expression (11):

$$
\mathrm{d}\left(Q-Q_{0}\right) / \mathrm{d} x=-h(K, I, \ldots)\left[\left(Q-Q_{0}\right)^{1+\alpha}\right] / \alpha
$$

ce qui donne par intégration :

$$
\frac{1}{\left(Q_{2}-Q_{0}\right)^{\alpha}}-\frac{1}{\left(Q_{1}-Q_{0}\right)^{\alpha}}=\left(x_{2}-x_{1}\right) h(K, I, \ldots)
$$

ou encore :

$$
\frac{Q_{2}-Q_{0}}{Q_{1}-Q_{0}}=\frac{1}{\left[1+\left(x_{2}-x_{1}\right) h(K, I, \ldots)\left(Q_{1}-Q_{0}\right)^{\alpha}\right]^{1 /}} \alpha
$$

On peut poser $h(K, I, \ldots)\left(Q_{1}-Q_{0}\right)^{\alpha}=1 / D_{5}$

avec $D_{5}$ ayant la dimension d'une longueur.

L'expression (12) devient, en notant que $x_{2}-x_{1}=L$ :

$$
\frac{Q_{2}-Q_{0}}{Q_{1}-Q_{0}}=\frac{1}{\left(1+L / D_{5}\right)^{1 / \alpha}} .
$$

Nous allons abandonner le modèle (11) et modifier le résultat précédent pour construire empiriquement un modèle plus précis mais non généralisable à un pas d'espace quelconque. On peut introduire à cet effet un paramètre $\beta$ supplémentaire tel que $\beta \in 0,1$ et :

$$
\frac{\left(Q_{2}-Q_{0}\right)-\beta\left(Q_{2}-Q_{0}\right)}{\left(Q_{1}-Q_{0}\right)-\beta\left(Q_{2}-Q_{0}\right)}=\frac{1}{\left(1+L / D_{5}\right)^{1 / \alpha}}
$$

ce qui donne l'expression (13):

$$
Q_{2}=Q_{0}+\frac{Q_{1}-Q_{0}}{\beta+(1-\beta)\left(1+L / D_{5}\right)^{1 / \alpha}} .
$$

Inversement cette expression (13) permet de définir $D_{5}$ d'après les observations de $Q_{1}$ et de $Q_{2}$, et de caler un modèle explicatif pour $D_{5}$ en s'affranchissant des hypothèses de départ, inutiles puisque l'on a choisi de sélectionner un modèle correspondant à un pas d'espace bien défini. Ce modèle s'écrit :

$$
\begin{aligned}
D_{5}=a_{0} K^{a 1}\left(I+a_{5}\right)^{a 2}\left[P _ { 1 } \left(Q_{1}\right.\right. & \left.\left.-Q_{0}\right) / W+a_{6}\right]^{a 3} \times \\
& \times\left[\left(Q_{1}-Q_{0}\right) / W\right]^{a 4} .
\end{aligned}
$$

\subsubsection{Temps de transfert de la pointe de crue}

Reprenons le modèle (10) :

$$
\mathrm{d} T / \mathrm{d} x=g(K, I, \ldots)
$$

on peut expliciter $g$ de la façon suivante:

$$
g(K, I, \ldots)=k(K, I, \ldots) /\left[\left(Q-Q_{0}\right) / W\right]^{\sigma}
$$

et d'après (13), on a :

$$
\begin{aligned}
\left(\frac{Q-Q_{0}}{W}\right)^{\sigma} & =\left(\frac{Q_{1}-Q_{0}}{W}\right)^{\sigma} \times \\
& \times \frac{1}{\left\{\beta+(1-\beta)\left[1+\left(x-x_{1}\right) / D_{5}\right]^{1 / \alpha}\right\}^{\sigma}} .
\end{aligned}
$$

Ce qui donne:

$$
\begin{aligned}
\mathrm{d} T / \mathrm{d} x=\frac{k(K, I, \ldots)}{\left[\left(Q_{1}-Q_{0}\right) / W\right]^{\sigma}} \times \\
\quad \times\left\{\beta+(1-\beta)\left[1+\left(x_{2}-x_{1}\right) / D_{5}\right]^{1 / \alpha}\right\}^{\sigma} .
\end{aligned}
$$

En posant

$$
\frac{k(K, I, \ldots)}{\left[\left(Q_{1}-Q_{0}\right) / W\right]^{\sigma}}=1 / C_{5}
$$

il vient :

$$
\mathrm{d} T / \mathrm{d} x=\frac{1}{C_{5}}\left\{\beta+(1-\beta)\left[1+\left(x_{2}-x_{1}\right) / D_{5}\right]^{1 / \alpha}\right\}^{\sigma}
$$

soit :

$$
C_{5} T=\int_{0}^{L}\left[\beta+(1-\beta)\left(1+x / D_{5}\right)^{1 / \alpha}\right]^{\sigma} \mathrm{d} x .
$$

La relation (15) permet de calculer $C_{5}$ en fonction de $T$ et donc de caler un modèle explicatif pour $C_{5}$ :

$$
\begin{aligned}
C_{5}=b_{0} K^{b_{1}}\left(I+b_{5}\right)^{b_{2}}\left[P _ { 1 } \left(Q_{1}\right.\right. & \left.\left.-Q_{0}\right) / W+b_{6}\right]^{b_{3}} \times \\
& \times\left[\left(Q_{1}-Q_{0}\right) / W\right]^{b_{4}}
\end{aligned}
$$

avec $b_{4}=\sigma$.

\section{Explicitation du modèle simplifié de propagation}

La simplification résultant de la non-influence de la forme de la crue sur les composantes de la propagation nous autorise à refaire des simulations pour mieux détailler l'influence des paramètres effectivement 


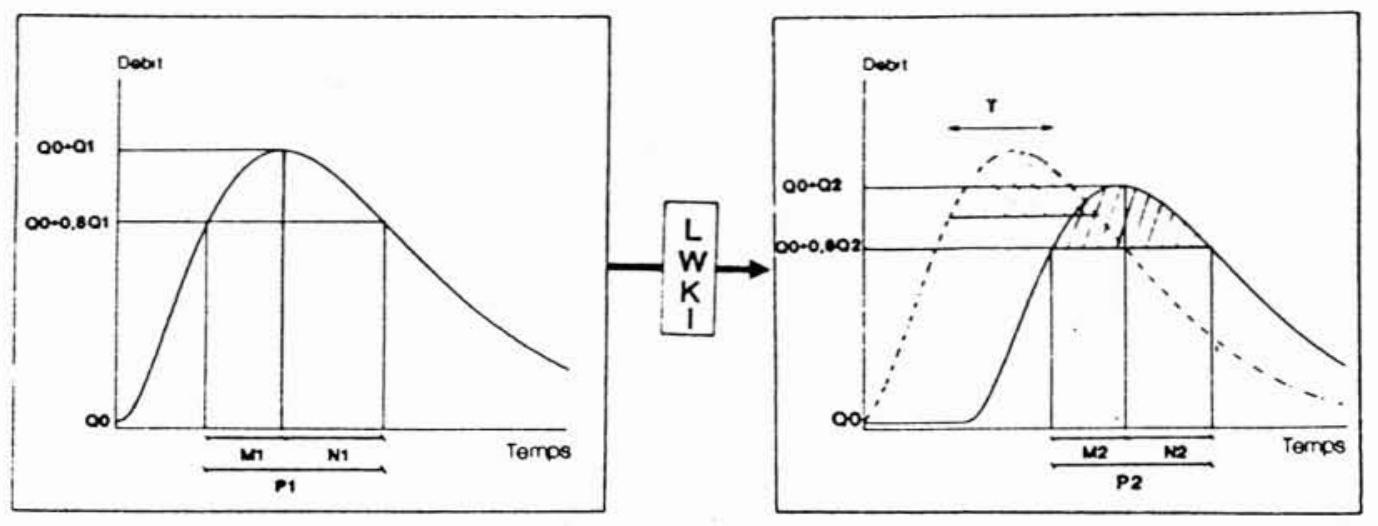

5. Le modèle simplifié de propagation.

influents. Nous avons refait les simulations avec le jeu de variables suivantes :

$$
\begin{aligned}
& K \quad=15 ; 25 ; 35 \text { et } 45 \mathrm{~m}^{1 / 3} \mathrm{~s}^{-1} ; \\
& I \quad=0,00005 ; 0,00015 ; 0,00045 \text { et } 0,00135 \mathrm{~m} / \mathrm{m} ; \\
& Q / W=1 ; 2 ; 4 \text { et } 8 \mathrm{~m}^{2} \mathrm{~s}^{-1} ; \\
& Q_{0} / W=0,2 \mathrm{~m}^{2} \mathrm{~s}^{-1} ; \\
& L \quad=5000 \mathrm{~m}, \text { pas d'espace fixe du modèle. }
\end{aligned}
$$

Ces nouvelles simulations nous permettent de caler les modèles (14) et (16). Les coefficients additifs ont été calés par tâtonnement, et les exposants par régression multiple. On a trouvé $\alpha=2 ; \beta=0,85$ et :

$$
\begin{array}{llll}
a_{0}=0,95 & a_{1}=1,27 & a_{2}=1,59 & a_{3}=1,64 \\
a_{4}=-1,09 & a_{5}=10^{-4} & a_{6}=0 & \\
b_{0}=205 & b_{1}=0,23 & b_{2}=-0,09 & \\
b_{3}=-0,66 & b_{4}=0,66 & b_{5}=10^{-4} & b_{6}=1,1 \times 10^{4} .
\end{array}
$$

On vient donc d'établir un système simple pour la propagation d'une pointe de crue le long d'un bief de caractéristiques données. La méthode consiste à décomposer le bief en tronçons de $5000 \mathrm{~m}$ de longueur, le dernier étant le seul à avoir éventuellement une longueur plus faible. Pour chaque tronçon de longueur $L \leqslant 5000 \mathrm{~m}$ la propagation se présente comme illustré par la figure 5 :

Les éléments du système sont les suivants :

- crue amont, décrite par $Q_{1}$ et $P_{1}$, respectivement le débit de pointe et la durée de la crue pour la partie de l'hydrogramme dépassant $80 \%$ du débit de pointe (et subsidiairement la décomposition de $P_{1}$ en $M_{1}$ et $N_{1}$, correspondant à la montée et à la décrue, respectivement). Le débit de base, $Q_{0}$, est neutre sur la propagation ;

- les tronçons de cours d'eau, de longueur $L \leqslant 5000 \mathrm{~m}$, largeur $W$, coefficient de STRICKLER $K$ et pente $I$.
Les trois variables à déterminer, $Q_{2}, P_{2}$, et $T$, sont obtenues par trois équations algébriques, utilisant les variables intermédiaires $C_{5}$ et $D_{5}$, dont le calage a donné les expressions suivantes :

$$
\begin{aligned}
C_{5}=200 K^{0,23}\left(I+10^{-4}\right)^{-0,09} \times \\
\times\left[P_{1}+\left(1,1 \times 10^{4} W\right) /\left(Q_{1}-Q_{0}\right)\right]^{-0,66} \\
D_{5}=0,95 K^{1,27}\left(I+10^{-4}\right)^{1,59} \times \\
\quad \times P_{1}^{1,64}\left[\left(Q_{1}-Q_{0}\right) / W\right]^{0,55} .
\end{aligned}
$$

Les équations algébriques sont alors :

$$
\begin{aligned}
Q_{2} & =Q_{0}+\frac{Q_{1}-Q_{0}}{0,85+0,15\left(1+L / D_{5}\right)^{0.5}} \\
P_{2} & =P_{1}\left(Q_{1}-Q_{0}\right) /\left(Q_{2}-Q_{0}\right) \\
T & =\frac{D_{5}}{C_{5}}\left\{0,72 L / D_{5}+0,21\left[\left(1+L / D_{5}\right)^{1,33}-1\right]\right\}
\end{aligned}
$$

L'expression (21) découle d'une estimation simplifiée de l'intégrale apparaissant dans (15). Dans ces équations $L=5000 \mathrm{~m}$, sauf peut-être pour le tronçon terminal du bief étudié.

Rappelons que dans toutes ces équations les unités sont celles du système S.I. L'erreur standard de ce modèle est de $6 \%$ environ pour $Q_{2}$ et $23 \%$ pour $T$.

On rappelle aussi que la forme de la crue $(M / N)$ n'intervient pas de façon très sensible dans le calcul de $Q_{2}$ et de $T$. Cependant, si on connaît la forme de la crue à l'amont grâce à une identification séparée de $M_{1}$ et de $N_{1}$, on peut en déduire la déformation de la crue, tout au long d'un tronçon, et par suite, calculer $M_{2}$ et $N_{2}$ par les expressions (22) et (23) ci-après :

$$
\begin{aligned}
& M_{2}=\frac{\left(M_{1}+N_{1}\right)\left(Q_{1} / Q_{2}\right)}{M_{1}+N_{1}\left(Q_{1} / Q_{2}\right)^{0,6}} M_{1} \\
& N_{2}=\left(M_{2} / M_{1}\right)\left(Q_{1} / Q_{2}\right)^{0,6} N_{1} .
\end{aligned}
$$


Cette opération est à répéter autant de fois qu'il y a de tronçons de $5000 \mathrm{~m}$ dans le bief étudié.

Le modèle proposé est très simple et d'une approximation suffisante pour dégrossir le problème de la propagation au stade d'un avant-projet.

\section{Application numérique}

Dans le but d'illustrer l'applicabilité du modèle proposé, on l'a utilisé ci-après sur un cas réel. La rivière Jacui, située au sud du Brésil, présente des éléments qui paraissent a priori favorables à l'étude de la propagation. En effet, d'après (I.P.H., 1983) et (ZAMANILLO et TUCCI, 1985), on dispose d'hydrogrammes correspondant à des lâchures de barrage, observés au long d'un tronçon de $35600 \mathrm{~m}$, avec des contributions intermédiaires négligeables. On dispose aussi de profils en long et en travers. Les caractéristiques du tronçon à l'étude sont présentées au tableau 1.

Pour l'application du modèle, on a travaillé avec 4 événements dont les caractéristiques apparaissent au
Tableau 1. Caractéristiques du tronçon

\begin{tabular}{|c|c|c|c|c|}
\hline Bief & $L(\mathrm{~m})$ & $W(\mathrm{~m})$ & $I$ & $K\left(\mathrm{~m}^{1 / 3} \mathrm{~s}^{-1}\right)$ \\
\hline I & 9000 & 50 & $1,6910^{-3}$ & 35 \\
\hline II & 12600 & 60 & $0,6710^{-3}$ & 35 \\
\hline III & 3600 & 70 & $1,9410^{-3}$ & 40 \\
\hline IV & 10400 & 80 & $0,5610^{-3}$ & 40 \\
\hline
\end{tabular}

tableau 2. Les résultats obtenus pour l'évaluation du débit aval et du décalage, ainsi que les erreurs commises sont présentés dans le tableau 2. Les unités sont toujours celles du S.I.

L'utilisation d'un modèle classique complet nous conduirait certainement à des résultats plus précis, mais on voit que le modèle proposé présente une performance qui peut être acceptable pour un dégrossissage préalable.

Tableau 2. Données utilisées et résultats obtenus

\begin{tabular}{|c|c|c|c|c|c|c|c|c|c|}
\hline \multirow{3}{*}{ Crue } & \multicolumn{3}{|c|}{ Données amont } & \multicolumn{6}{|c|}{ Données aval } \\
\hline & \multirow{2}{*}{$\begin{array}{c}Q_{0} \\
m^{3} s^{-1}\end{array}$} & \multirow{2}{*}{$\begin{array}{c}Q_{1} \\
m^{3} s^{-1}\end{array}$} & \multirow{2}{*}{$\begin{array}{l}P_{1} \\
\mathrm{~s}\end{array}$} & \multicolumn{3}{|c|}{$Q_{2}\left(\mathrm{~m}^{3} \mathrm{~s}^{-1}\right)$} & \multicolumn{3}{|c|}{$T(\mathrm{~s})$} \\
\hline & & & & observé & calculé & erreur & observé & calculé & erreur \\
\hline 1 & 140 & 575 & 3500 & 396 & 377 & $-5 \%$ & 14300 & 14100 & $-1 \%$ \\
\hline 2 & 150 & 428 & 4000 & 298 & 300 & $1 \%$ & 15100 & 16600 & $10 \%$ \\
\hline 3 & 130 & 621 & 4500 & 452 & 445 & $-2 \%$ & 11300 & 14600 & $29 \%$ \\
\hline 4 & 75 & 505 & 5700 & 401 & 377 & $-6 \%$ & 10300 & 16200 & $57 \%$ \\
\hline
\end{tabular}

\section{Conclusion}

L'étude effectuée permet donc de faire une pré-estimation rapide de la propagation d'une pointe de crue. La méthode employée repose sur des simulations faites avec le système complet d'équations de BARRÉ DE SAINT VENANT, bien qu'appliqué à une situation schématique.

Les simulations effectuées sur un nombre conséquent de biefs et de crues permettent la généralisation de la méthode, et donc assurent son applicabilité à une gamme importante de situations qui se présentent dans la pratique de l'hydrologie.

Pour des estimations directes, les erreurs du modèle sont acceptables et les estimations sur des comparaisons peuvent être encore plus intéressantes. C'est ainsi que les relations établies permettent de mieux apprécier le rôle des différentes caractéristiques physiques, des cours d'eau et des crues, sur le phénomène de propagation.
Une condition aval très particulière ou une précision demandée plus fine que celle annoncée nécessitent le recours à la modélisation directe.

En particulier, la méthode pourrait être utile pour des études préliminaires d'impacts d'aménagements de cours d'eau. En effet, sachant que les paramètres caractéristiques des cours d'eau apparaissent de façon explicite dans les résultats, l'évaluation de l'impact d'un aménagement peut être dégrossie immédiatement.

\section{Remerciements}

Les auteurs sont reconnaissants à Rémy Pochat, directeur scientifique de l'Ecole nationale de Génie rural, des Eaux et des Forêts et directeur du CERGRENE pour ses conseils avisés. 


\section{Références}

Аввот M.B., (1979) : Computational hydraulics. Elements of the theory of free surface flows; Pitman Publishing limited.

CEMAGREF, Division hydrologie et hydraulique d'ANTONY (1985) : "Atténuation des crues dans un bief large" ; Note d'information technique $n^{*} 3$, cahier 57,3 pages.

CHOw V.T. (1959): Open channel hydraulics; McGraw Hill Book Company, $22^{\mathrm{e}}$ édition, 1974.

Cunge J.A., Holly J.F.M., Verwey A. (1980): Practical aspects of computational river hydraulics ; Pitman Publishing limited.

I.P.H., Instituto de Pesquisas Hidraulicas (1983): Estudos hidrodinamicos do Rio Jacui: Rapport technique IPHUFRGS.

Pochat R. (1980): Hydraulique à surface libre. Ecole d'été de mécanique des fluides.

Pridal D.B., Wesley P.J. (1989) : " Routing procedure for ungaged channels ". Journal of water resources planning and management, vol. 115, $\mathrm{n}^{\circ} 1$, pages 108 à 121 .

Tingsanchali T., LaL N.K. (1988): "Subsidence of flood waves in overbank flow areas ". Journal of hydraulic research, vol. $26, \mathrm{n}^{\circ} 5$, pages 585 à 597.

TucC1 C.E.M. (1986), Modelos matematicos en hidrologia $e$ hidraulica. Associaçao brasileira de recursos hidricos.

Zamanillo E., Tucci C.E.M. (1987): Simulaçao de escoamento por Muskingum-Cunge com parametros variaveis. Actes du VII Congrès brésilien de ressources en eau. 